\title{
Elementos prismáticos paramétricos tridimensionales utilizados para edificación virtual
}

\section{Using Tridimensional Parametric Prismatic Elements in Virtual Building}

\author{
Zaragoza-Grifé J.N. \\ Facultad de Ingeniería \\ Universidad Autónoma de Yucatán \\ E-mail:zgrife72@uady.mx
}

\author{
Pech-Pérez J.G. \\ Facultad de Ingeniería \\ Universidad Autónoma de Yucatán \\ E-mail:pperez@uady.mx
}

Información del artículo: recibido: junio de 2009, aceptado: junio de 2010

\section{Resumen}

En este artículo se muestran el desarrollo y la utilización de los Elementos Prismáticos Paramétricos Tridimensionales (EPPT) para la edificación virtual. Estos elementos se utilizan como parte esencial de un software programado por los autores en la Facultad de Ingeniería de la UADY, que tiene como objetivo edificar virtualmente proyectos de construcción utilizando BIM (Building Information Model). Los elementos matemáticos que sustentan su desarrollo y utilización se formulan con nivel de detalle geométrico y algebraico. También se muestra el desarrollo del sistema de referencia para la manipulación de los EPPT, junto con el procedimiento para el cálculo de propiedades geométricas de los EPPT para su utilización en la cuantificación, tales como: área, volumen y longitud. Como resultados, además del desarrollo de los EPPT, se presentan algunas pantallas de un software ya desarrollado que utiliza los conceptos aquí presentados, se discuten algunas cuestiones del desarrollo y se plantean posibles respuestas como solución basadas en una técnica de inteligencia artificial (IA). Entre las conclusiones más importantes, los autores mencionan que en la mayoría de las herramientas comerciales los procesos funcionan como cajas negras y que aportan poco al conocimiento para mejorar el desarrollo tecnológico en el tema de edificación virtual. Además, los autores proponen el material expuesto en este artículo como material didáctico para cursos de matemáticas aplicadas a nivel licenciatura en las carreras de ingeniero civil o constructor, particularmente en cursos de cálculo vectorial.

\section{Descriptores}

- edificación virtual

- Building Information Model (BIM)

- CAD

- Fuzzy Logic

- Software para construcción 


\begin{abstract}
The development and use of Tridimensional Parametric Prismatic Elements (TPPE) is introduced in this paper. Such elements are used as basis for the software specifically programmed by the authors in the Faculty of Engineering of Autonomous University of Yucatan, (FIUADY). The main purpose for this software is the virtual building of construction projects using BIM (Building Information Model). The mathematics and geometry used in the development of TPPE are show in detail. Also, the development of the reference system used to manipulate TPPE is presented. Procedures are shown for the calculation of geometric properties such as area, volume and length of an arbitrary TPPE. As a result of this work, we present a few software screenshots and a discussion about of development of the concepts for this paper. Some recommendations are made by the authors about using Artificial Intelligence (AI) for solving geometric intersections among TPPE. One of the most important conclusions of this work is, as mentioned, that most software tools available in the market today work like a black box and provide little knowledge contribution in order to improve BIM technology. Furthermore, the authors recommend that these topics can be used for educational purposes in undergraduate courses of Civil or Construction Engineering, particularly in Vectorial Calculus.
\end{abstract}

\section{Introducción}

La edificación virtual ha venido tomando auge en los últimos años, gracias a los avances en las tecnologías de información y el potencial creciente del hardware (Revit, 2008). Así como ahora es difícil concebir que se dibujen planos constructivos a mano, ya que en la actualidad se utilizan sistemas CAD (Computer Aided Design), pronto lo será pensar que las construcciones no se edifiquen primero de manera virtual (Nassar et al., 2002). Esto conlleva muchas ventajas muy por encima del dibujo CAD tradicional. Entre las ventajas más sobresalientes, existen aquellas que permitirán prever problemas constructivos antes de que ocurran en la realidad. Además de la visualización tridimensional, tanto la cuantificación como el costeo se obtienen de manera directa. Otro aspecto importante es que al modificar algún parámetro todo el modelo virtual puede ser actualizado de manera automática sin perder su consistencia topológica (Sacks et al., 2003).

Cabe mencionar que se han realizado investigaciones para que los elementos constructivos reales posean sensores que de manera automática realicen el vaciado de información proporcionando datos acerca de su localización en el lugar de la obra y se pueda digitalizar el modelo de la construcción para llevar un adecuado control sobre cambios y modificaciones del mismo (Suter et al., 2007).

Así mismo, el impacto de la edificación virtual en la compartición de información entre equipos de trabajo, conformados por ingenieros y arquitectos ha cobrado una importancia tal que se han desarrollado esfuerzos comunes para implantar por medio de servidores web soluciones que permiten hacer más eficientes estos procesos (Chen et al., 2005)

En México, ya se hace uso de paquetería de software comercial de firmas reconocidas, asociada con la edificación virtual (ArchiCAD, 2009). También se han realizado notables esfuerzos para la cuantificación, costeo y control de obra por medio del uso de la computadora y planos en formato electrónico (AllPlan, 2009) y (NeoDataCAD, 2009).

Sin embargo, en su mayoría, los paquetes computacionales mencionados no son adaptables al tipo de construcción que se lleva a cabo en México. Por lo que no es conveniente que los profesionales dedicados al diseño y a la construcción sean simples usuarios de paquetes comerciales, sino que participen en el desarrollo de herramientas, producto de investigaciones que se adapten a las necesidades particulares de cada uno. Por ello, en este artículo se propone una solución original para el desarrollo de un sistema computacional adaptable, que permita realizar la edificación virtual para las construcciones típicas de la región.

\section{Metodología}

Los Elementos Prismáticos Paramétricos Tridimensionales

Los elementos Prismáticos Paramétricos Tridimensionales (EPPT) que se muestran en este artículo como 
parte central de la propuesta, tienen como base las entidades geométricas y los objetos sólidos primarios presentados en (Kunigahalli, 1997), los cuales son prismas regulares formados por seis caras, cada una de las cuales tiene cuatro aristas definidas mediante dos vértices. Los autores del presente trabajo se permitieron realizar algunas adecuaciones para poder representar y manipular los EPPT de manera que se puedan implementar en la computadora. Entre otras aportaciones para la manipulación de objetos sólidos primarios se encuentran la de (Talbot, 2007), el cual propone un sistema generativo sensible al contexto para el dimensionamiento y colocación de los elementos constructivos en el dibujo, manejando las intersecciones y las proyecciones con los elementos circundantes en la vecindad del dibujo.

\section{Sistema de referencia en planta}

Para la utilización de los EPPT, se tomará el sistema de referencia utilizado denominado sistema de ejes constructivos o de trazo en planta. Este sistema por lo general se encuentra representado en la mayoría de los planos ejecutivos para edificación. Se utilizan ampliamente para referenciar las proyecciones en planta de los diferentes elementos de edificación que forman parte del proyecto. Por lo general, este sistema de ejes se utiliza para los planos de proyección en planta, aunque en ocasiones también se les encuentra en forma de referencia vertical en los planos de corte y alzado.

Dependiendo de las particularidades del proyecto, los ejes pueden ser: ortogonales, inclinados, circulares y por último, de formas muy específicas tales como elípticos, parabólicos, etc.

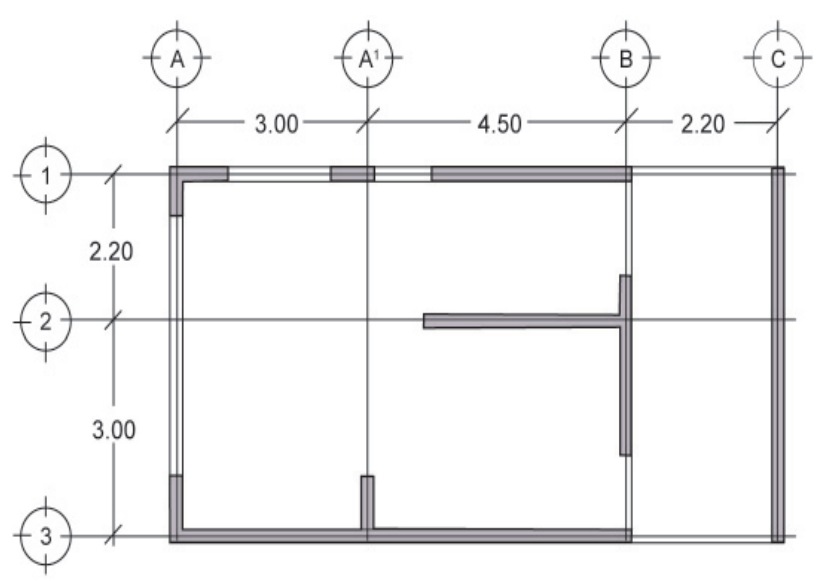

PLANTA ARQUITECTÓNICA

Figura 1. Planta arquitectónica típica con referencias a ejes
Cada eje debe contar con un identificador único, este identificador por lo general es una letra ó un número. Es común encontrar en los dibujos de los planos ejecutivos que los ejes verticales tienen como identificador único una letra y los horizontales un número (figura 1). Es posible también encontrar identificadores para los ejes con la misma letra de otro eje ya existente, pero con uno o más apóstrofos precediendo a la letra o al número. Por lo general, estos ejes fueron agregados a los planos posteriormente, ya sea por cambios en el proyecto o por una simple omisión de los mismos.

Entonces, para el caso de los ejes rectilíneos es necesario para su representación contar con al menos dos puntos que no necesariamente son los extremos del eje y un nombre de identificación válido único. Es de observarse que los puntos obedecen al sistema de referencia cartesiano rectangular (figura 2).

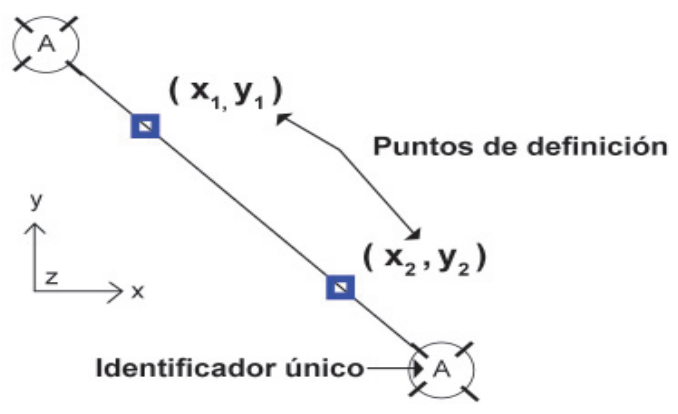

Figura 2. Eje inclinado con sus elementos que lo definen

Para representar un eje circular es necesario conocer el radio y la ubicación del centro del círculo. Cabe mencionar, que una variante importante de eje de tipo circular es el que tiene forma de arco circular. Puede ser que en el dibujo del plano, eventualmente sea necesario usar una parte del círculo, por lo que para el caso del arco, es necesario definir el ángulo de origen y el ángulo de terminación, los cuales están referenciados a un sistema polar local con origen en el centro del arco, en donde el sentido positivo de los ángulos ocurre en el sentido contrario a las manecillas del reloj. Se mide a partir del eje positivo ' $x$ ' de referencia local (figura 3).

De manera similar, se puede definir cualquier trayectoria para los ejes, sólo es necesario conocer los parámetros geométricos que los definen.

Por ahora, se ha tratado la definición de los ejes y sus propiedades particulares; sin embargo, la mayor importancia es evidente cuando se tiene un sistema de estos ejes. En el sistema de ejes se da un conjunto de intersecciones que servirán para ubicar a los EPPT (figura 4). 


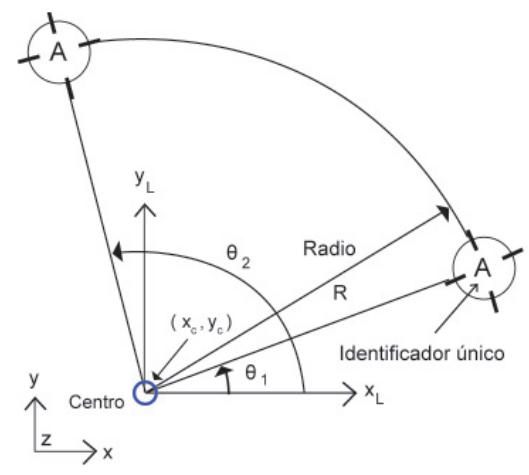

Figura 3. Eje en forma de arco con sus elementos que lo definen
La ubicación en planta de los EPPT se define mediante dos y sólo dos puntos de intersección provenientes del sistema de ejes. Cabe mencionar que estos puntos de intersección se dan en el plano cartesiano con $\mathrm{z}=0$. A este par de puntos P1 y P2 se les denominará puntos de inserción, respectivamente.

También cada uno de los EPPT que forman parte de la edificación virtual tiene dos conjuntos de parámetros, el primero $\left\{\mathrm{L}, \mathrm{C}_{1}, \mathrm{C}_{2} \mathrm{y} \mathrm{T}_{\mathrm{L}}\right\}$ y el segundo $\left\{\mathrm{B}, \mathrm{B}_{1}, \mathrm{~B}_{2} \mathrm{y}\right.$ $\mathrm{T}_{\mathrm{B}}$ \} (figura 5). Cabe mencionar que la propuesta actual es que el EPPT en planta sea ortogonal. Aunque más adelante se discutirá la manera de representar las intersecciones con otros EPPT en el modelo.

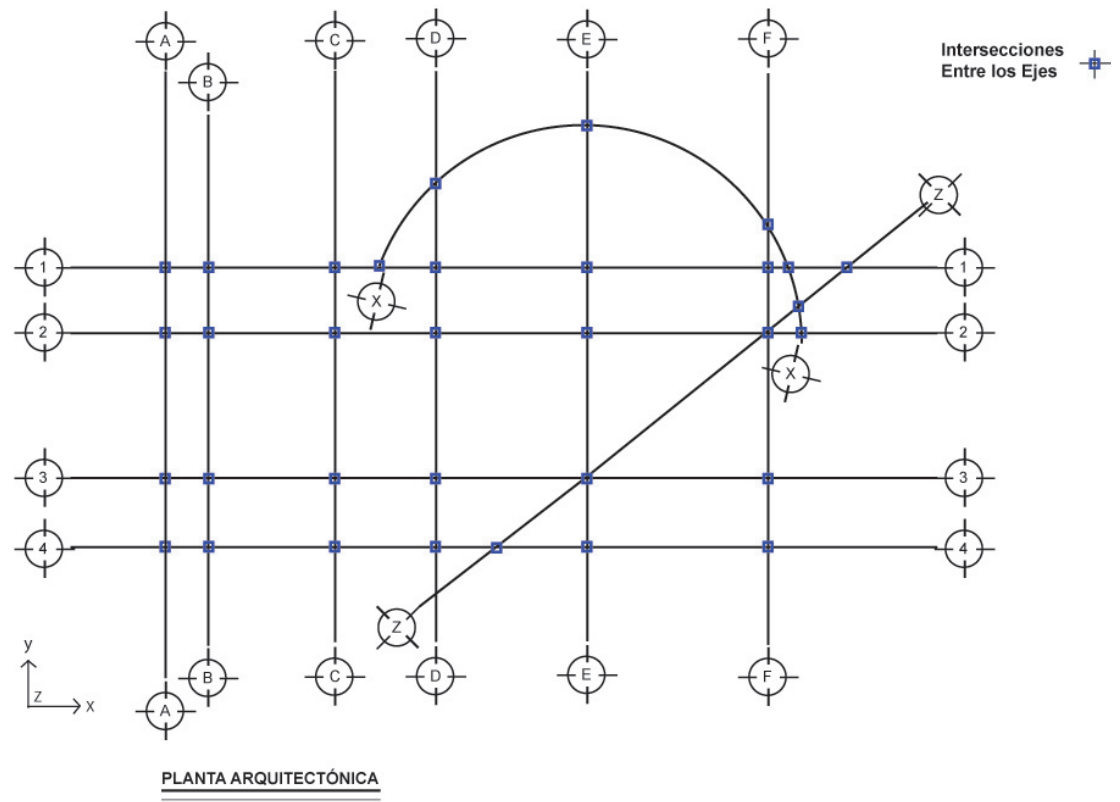

Sistema de ejes en el plano

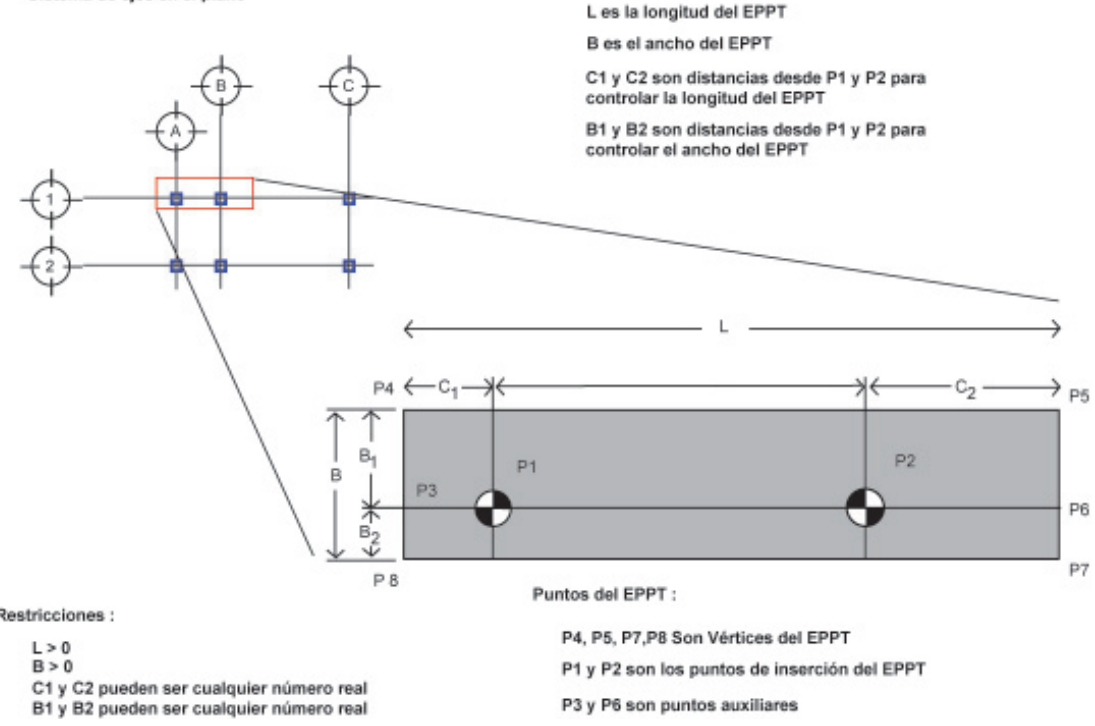

Figura 4. Planta arquitectónica de ejemplo con las intersecciones entre los ejes marcadas
Figura 5. Descripción de un Elemento Prismático Paramétrico Tridimensional 
Es aquí donde las matemáticas intervienen con detalle. Para poder representar la proyección en el plano del sistema de ejes del EPPT es necesario calcular el conjunto de puntos $\{\mathrm{P} 3, \mathrm{P} 4, \mathrm{P} 5, \mathrm{P} 6, \mathrm{P} 7, \mathrm{P} 8\}$. El cálculo de las coordenadas de estos puntos dependerá de los parámetros del EPPT (tabla 1).

La metodología para la obtención del conjunto de puntos que definen al EPPT se detalla a continuación:

Pasos 1 a 3: Cálculos preliminares

Paso 1. Los puntos de inserción P1 y P2 son definidos.

Paso 2. Los parámetros $\left\{\mathrm{L}, \mathrm{C}_{1}, \mathrm{C}_{2}\right.$ y $\left.\mathrm{T}_{\mathrm{L}}\right\}$ y $\left\{\mathrm{B}, \mathrm{B}_{1}, \mathrm{~B}_{2} \mathrm{y} \mathrm{T}_{\mathrm{B}}\right\}$ son definidos.

Paso 3. Calcular el vector unitario entre P1 y P2 al cual se le denominará $V U n i$. Para calcular el vector unitario se utiliza la ecuación (1).

VUni $=\frac{\left\langle\left(P 2_{x}-P 1_{x}\right),\left(P 2_{y}-P 1_{y}\right),\left(P 2_{z}-P 1_{z}\right)\right\rangle}{\sqrt{\left(P 2_{x}-P 1_{x}\right)^{2}+\left(P 2_{y}-P 1_{y}\right)^{2}+\left(P 2_{z}-P 1_{z}\right)^{2}}}$

Pasos 4 a 5: Obtención de los puntos auxiliares P3 y $\mathrm{P} 6$

Para poder determinar las coordenadas de los puntos que conforman la proyección en el plano XY del EPPT se han utilizado dos ecuaciones; la ecuación (2) que permite encontrar las coordenadas de un punto PA conocido, otro punto PB ubicado a una determinada distancia $\mathrm{D}$ y con el vector de dirección $\mathrm{V}$ definido entre los dos puntos conocidos.

$$
\begin{aligned}
& P A_{x}=P B_{x}+D \cdot V_{x} \\
& P A_{y}=P B_{y}+D \cdot V_{y} \\
& P A_{z}=P B_{z}+D \cdot V_{z}
\end{aligned}
$$

Por otro lado, se utiliza también la ecuación (3) que permite encontrar las coordenadas de un punto PC para el cual su proyección perpendicular a una distancia $\mathrm{D}$ cae sobre un punto conocido $\mathrm{PB}$, el cual está alineado con un vector de dirección $\mathrm{V}$ (figura 7).

$$
\begin{aligned}
& P C_{x}=P B_{x}-D \cdot V_{y} \\
& P C_{y}=P B_{y}+D \cdot V_{x} \\
& P C_{z}=P B_{z}+D \cdot V_{z}
\end{aligned}
$$

Paso 4a. Si el parámetro $\mathrm{T}_{\mathrm{L}}$ es igual a 0, es decir: “Dado L y $C_{1}$ " o si el parámetro $T_{L}$ es igual a 2, es decir: "Dado $\mathrm{C}_{1} \mathrm{y} \mathrm{C}_{2}$ ", entonces el punto $\mathrm{P} 3$ es calculado utilizando el

\begin{tabular}{|c|c|c|}
\hline Parámetro & Descripción & Rango \\
\hline P1 & Punto de Inserción 1 & $\begin{array}{l}\text { Pareja ordenada de reales obtenida desde } \\
\text { una intersección de dos Ejes del Sistema } \\
\text { de Ejes }\end{array}$ \\
\hline P2 & Punto de Inserción 2 & $\begin{array}{l}\text { Pareja ordenada de reales obtenida desde } \\
\text { una intersección de dos Ejes del Sistema } \\
\text { de Ejes }\end{array}$ \\
\hline $\mathrm{L}$ & Longitud & Solo positivos diferentes de cero \\
\hline $\mathrm{C}_{1}$ & $\begin{array}{l}\text { Distancia medida entre P1 } \\
\text { y P3 }\end{array}$ & Todos los números reales \\
\hline $\mathrm{C}_{2}$ & $\begin{array}{l}\text { Distancia medida entre P2 } \\
\text { y P6 }\end{array}$ & Todos los números reales \\
\hline $\mathrm{T}_{\mathrm{L}}$ & Tipo de longitud utilizada & $\begin{array}{l}\text { Puede ser: } \\
0 \text { - Dado L y } C_{1} \\
1 \text { - Dado } L \text { y } C_{2} \\
2 \text { - Dado } C_{1} \text { y C }_{2}\end{array}$ \\
\hline B & Ancho & Solo positivos diferentes de cero \\
\hline $\mathrm{B}_{1}$ & $\begin{array}{l}\text { Distancia medida entre P3 } \\
\text { y P4 }\end{array}$ & Todos los números reales \\
\hline B2 & $\begin{array}{l}\text { Distancia medida entre P3 } \\
\text { y P8 }\end{array}$ & Todos los números reales \\
\hline $\mathrm{T}_{\mathrm{B}}$ & Tipo de ancho utilizado & $\begin{array}{l}\text { Puede ser: } \\
0 \text { - Dado B y } \mathrm{B}_{1} \\
1 \text { - Dado B y } \mathrm{B}_{2} \\
2 \text { - Dado } \mathrm{B}_{1} \text { y } \mathrm{B}_{2}\end{array}$ \\
\hline
\end{tabular}
valor de $\mathrm{P} 1$ y el valor del parámetro de distancia $\mathrm{C}_{1}$, seguidamente el punto $\mathrm{P} 6$ es calculado utilizando el valor obtenido de $\mathrm{P} 3$ y el valor del parámetro de distancia L. El cálculo de los puntos P3 y P6 es efectuado con la ecuación (2).

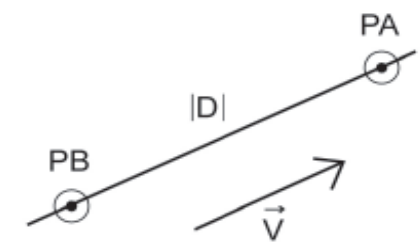

Figura 6. Representación gráfica de la ecuación (2)

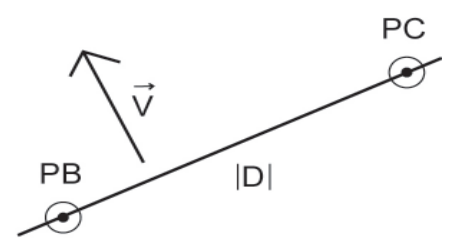

Figura 7. Representación gráfica de la ecuación (3)

Tabla 1. Descripción de Parámetros del Elemento Prismático Tridimensional 
Paso 4b. Por el contrario, si el parámetro $\mathrm{T}_{\mathrm{L}}$ es igual a 1 , es decir: "Dado $\mathrm{L}$ y $\mathrm{C}_{2}$ ” entonces primero el punto $\mathrm{P} 6$ es calculado utilizando el valor de P2 y el valor del parámetro de distancia $\mathrm{C}_{2}$, seguidamente se calcula el punto P3 utilizando el valor de P2 y el valor del parámetro de distancia L, pero con signo negativo. El cálculo de los puntos P3 y P6 es efectuado con la ecuación (2).

Paso 5: Obtención de los puntos P4, P5, P7 y P8

Dependiendo del valor del parámetro $\mathrm{T}_{\mathrm{B}^{\prime}}$, se tienen dos alternativas descritas en el Paso $5 a$ y el Paso 5b, respectivamente.

Paso 5a. Si el parámetro $\mathrm{T}_{\mathrm{B}}$ es igual a 0 , es decir: “Dado $\mathrm{B}$ y $\mathrm{B}_{1}$ ” o si el parámetro $\mathrm{T}_{\mathrm{B}}$ es igual a 2, es decir: “Dado $\mathrm{B}_{1}$ y $\mathrm{B}_{2}$ ", entonces el punto $\mathrm{P} 4$ es calculado utilizando el valor de $\mathrm{P} 3$ y el parámetro $\mathrm{B}_{1}$, el punto $\mathrm{P} 5$ es calculado utilizando el valor de $\mathrm{P} 6$ y el parámetro $\mathrm{B}_{1}$. El cálculo de los puntos P4 y P5 es efectuado con la ecuación (3). De manera análoga, el punto P7 es calculado utilizando el valor del punto P5 y el negativo del parámetro B; el punto P8 es calculado utilizando el valor del punto P4 y el negativo del parámetro B. Los puntos P7 y P8 son calculados con la ecuación (3).

Paso 5b. Por el contrario, si el parámetro $\mathrm{T}_{\mathrm{B}}$ es igual a 1, es decir: "Dado B y $\mathrm{B}_{2}$ ", entonces primero el punto $\mathrm{P} 7 \mathrm{es}$ calculado utilizando el valor del punto P6 y el negativo del valor del parámetro de distancia $\mathrm{B}_{2}$, utilizando la ecuación (3). El punto P5 es calculado utilizando el valor del punto $\mathrm{P7}$ y el valor de la distancia del parámetro B. El punto P8 es calculado utilizando el valor del punto P3 y el negativo del valor del parámetro de distancia $B_{2}$,
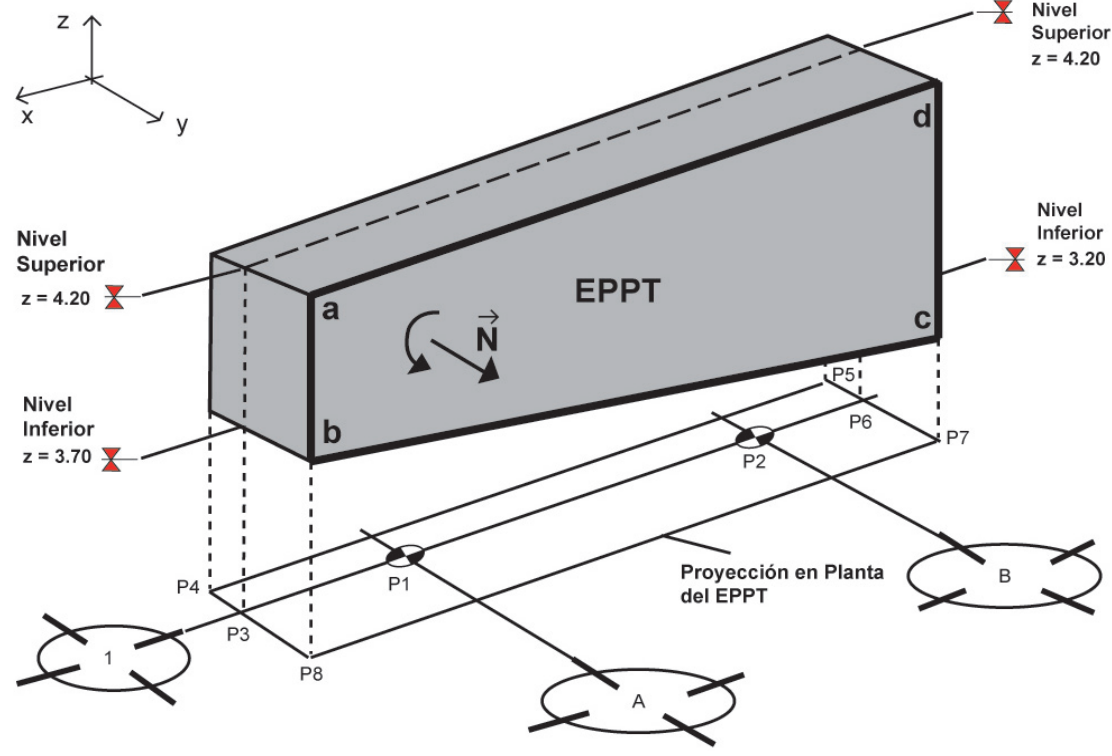

Z Nivel Superior
$z=4.20$

con la ecuación (3). Finalmente, se obtiene el valor del punto P4 con el valor del punto P8 y el valor del parámetro de distancia $B$, también con la ecuación (3).

\section{Sistema de referencia en alzado}

Para la representación en tres dimensiones del EPPT se utiliza el paradigma del nivel en la construcción. Matemáticamente este nivel está definido por un valor para la coordenada $\mathrm{z}$, la cual define ecuaciones de planos paralelos al plano XY (Plano $\mathrm{z}=0$ ).

El EPPT mantiene una proyección ortogonal en el nivel $z=0$. Se puede observar que para representarlo de manera tridimensional se necesita indicar a qué nivel inferior y superior estará asociado el EPPT en cada uno de sus extremos que están alineados con los puntos de inserción P1 y P2 (figura 8).

Cada una de las seis caras que dan forma al EPPT es definida mediante vértices espaciales que unen las aristas de las caras. Por ejemplo, la cara definida por el conjunto de vértices en el espacio $\{a, b, c, d\}$. Es importante que el cálculo de los vértices se haga en el sentido antihorario para hacer que el vector normal al plano abcd sea positivo. Es decir, si se toma el vector $b a$ y el vector $b c$ y se realiza el producto vectorial $b c \times b a$ se obtenga el vector normal $N$. Esto es con el objetivo de que en el paquete computacional donde se utilicen los EPPT, se pueda asignar un color a las caras y sean visibles a la hora de obtener la vista tridimensional final de todos los componentes.

Para dar una idea de cómo obtener las coordenadas de los puntos de la cara $\{$ abcd $\}$ se observa que los puntos a y b comparten las coordenadas $(\mathrm{x}, \mathrm{y})$ del punto P8; sin embargo, la coordenada en $\mathrm{z}$ del punto a y la coordenada en $z$ del punto $b$ tienen el valor de la $z$ de los niveles superior e inferior, asociados al extremo 1 del EPPT, respectivamente. De la misma manera, la coordenada en $z$ del punto $d$ y la coordenada en $\mathrm{z}$ del punto $\mathrm{c}$ tienen el valor de la $\mathrm{z}$ de los niveles superior e inferior, asociados al extremo 2 del EPPT, respectivamente (tabla 2, figura 9).

Figura 8. Representación tridimensional del EPPT 


\begin{tabular}{cll}
\hline Parámetro & \multicolumn{1}{c}{ Descripción } & \multicolumn{1}{c}{ Rango } \\
\hline N.S.1 & $\begin{array}{l}\text { Nivel superior asociado } \\
\text { al extremo 1 }\end{array}$ & $\begin{array}{l}\text { Un valor para z que puede tomar } \\
\text { cualquier valor real }\end{array}$ \\
N.I.1 & $\begin{array}{l}\text { Nivel inferior asociado } \\
\text { al extremo 1 }\end{array}$ & $\begin{array}{l}\text { Un valor para z que puede tomar } \\
\text { cualquier valor real }\end{array}$ \\
N.S.2 & $\begin{array}{l}\text { Nivel superior asociado } \\
\text { al extremo 2 } \\
\text { Nivel inferior asociado } \\
\text { al extremo 2 }\end{array}$ & $\begin{array}{l}\text { Un valor para z que puede tomar } \\
\text { cualquier valor real } \\
\text { Un valor para z que puede tomar } \\
\text { cualquier valor real }\end{array}$ \\
\hline
\end{tabular}

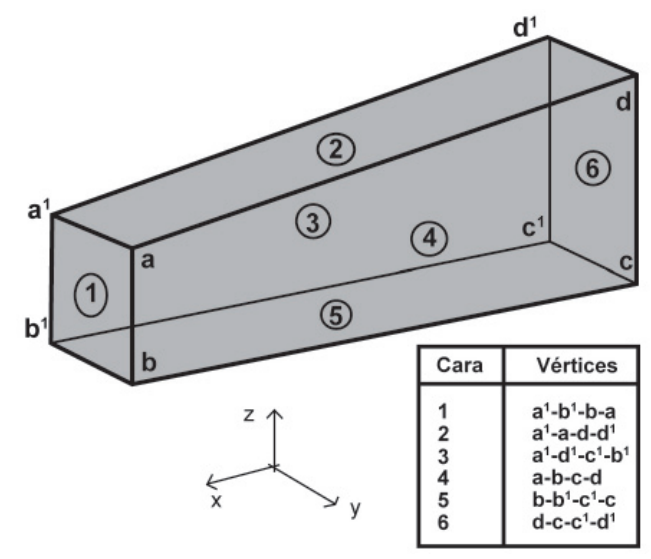

\section{Área}

Para la determinación del área de alguna de las caras se utilizan la magnitud del resultado de la operación del producto cruzado entre dos vectores definidos mediante dos aristas consecutivas. Es conocido del álgebra de vectores, que la magnitud del producto cruzado de dos vectores es igual al área de un paralelogramo, cuyos lados consecutivos tienen la misma magnitud que los vectores del producto respectivo. Pero por conveniencia, se puede obtener únicamente el área de un triángulo al dividir el resultado a la mitad. De la misma manera, se obtiene el área del otro triángulo que se forma con la multiplicación vectorial de los otros dos vectores que forman la cara. Entonces, el área de la cara es igual a la suma del área de los dos triángulos. La separación triangular es conveniente como solución general en caso de que los vértices de la cara formen un cuadrilátero irregular.

A manera de ejemplo, si se toma la cara número 4 con el conjunto de vértices $\{a, b, c, d\}$ entonces para determinar su área se utiliza la ecuación (4). Es evidente que los dos triángulos comparten un mismo lado que es una diagonal del cuadrilátero \{abcd\} (figura 10).

Area $=\frac{|\overrightarrow{a b} \times \overrightarrow{b c}|+|\overrightarrow{d a} \times \overrightarrow{d c}|}{2}$
Tabla 2. Descripción de parámetros del Elemento Prismático Tridimensional en su ubicación fuera del plano de proyección

Figura 9. Caras y vértices del Elemento Prismático Paramétrico Tridimensional

\section{Volumen}

Es conocido que para calcular el volumen de un tetraedro no regular cuyos vértices son A, B, C y D se puede utilizar la ecuación (5). Este método utiliza las coordenadas de los vértices y el cálculo de un determinante de grado cuatro.

$V=\frac{1}{6} \cdot\left|\begin{array}{cccc}1 & 1 & 1 & 1 \\ x_{A} & x_{B} & x_{C} & x_{D} \\ y_{A} & y_{B} & y_{C} & y_{D} \\ z_{A} & z_{B} & z_{C} & z_{D}\end{array}\right|$

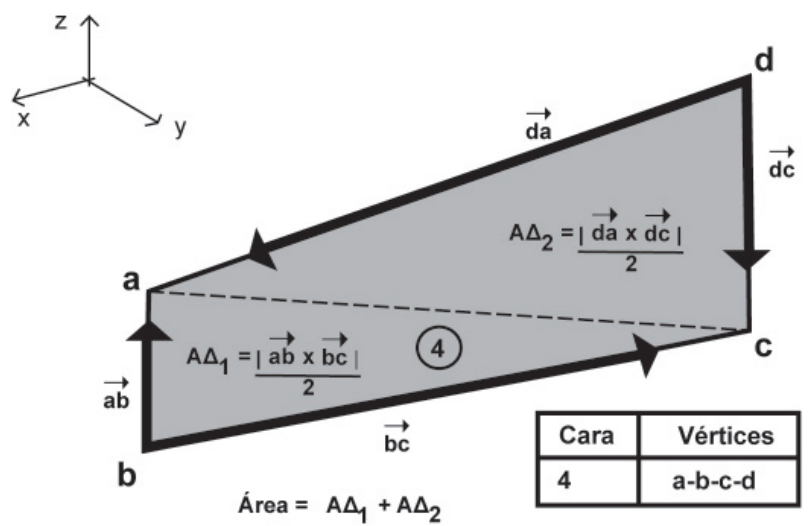

Figura 10. Área de la cara 4 de un Elemento Prismático Paramétrico Tridimensional 
Para calcular el volumen del EPPT, que para el caso más general pudiera no ser un paralelepípedo regular, de manera análoga como se formuló para el cálculo del área, se descompone el EPPT en cinco tetraedros, en donde su volumen se calcula con la ecuación (5) (figura 11). Los volúmenes de los tetraedros generales suman como resultado el volumen total del EPPT (tabla 3).

\section{Longitud}

En el caso del cálculo de la longitud, es necesario especificar qué distancia es la que se requiere. Por ejemplo, si se quiere la altura entonces bastaría con hacer la diferencia entre los valores de las coordenadas en z de los niveles superior e inferior de alguno de los extremos del EPPT. Por otra parte, si se desea obtener la longitud

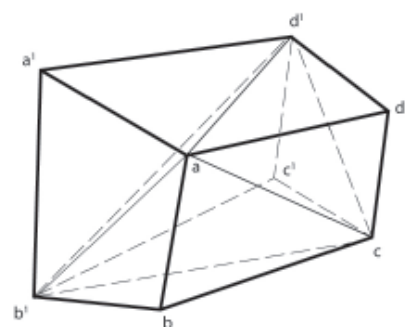

Figura 11. Descomposición del EPPT en cinco tetraedros para obtener su volumen

Tabla 3. Vértices de los cinco tetraedros que componen al EPPT

\begin{tabular}{cccc}
\hline Vértice A & Vértice B & Vértice C & Vértice D \\
\hline$b$ & $b^{\prime}$ & $a$ & $c$ \\
$d$ & $a$ & $d^{\prime}$ & $c$ \\
$a^{\prime}$ & $b^{\prime}$ & $a$ & $d^{\prime}$ \\
$c^{\prime}$ & $d^{\prime}$ & $c$ & $b^{\prime}$ \\
$a$ & $d^{\prime}$ & $c$ & $b^{\prime}$ \\
\hline
\end{tabular}

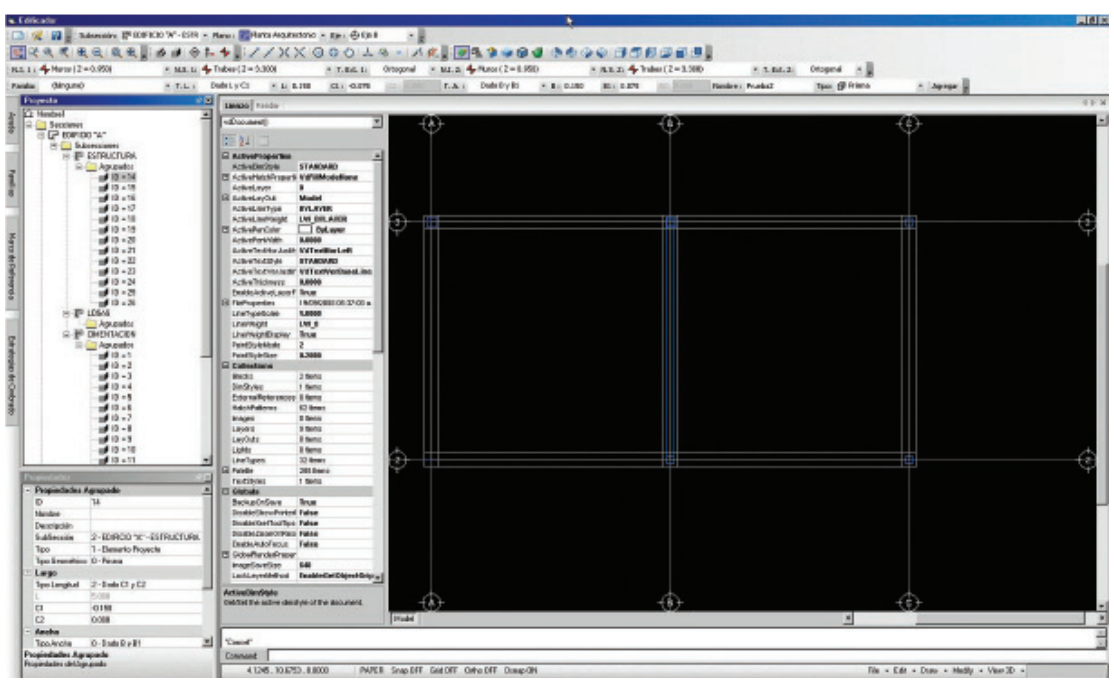

Figura 12. Pantallas de la herramienta desarrollada aplicando los EPPT's (continúa) y la anchura del EPPT se podría optar por escoger los valores que se tienen en la proyección en planta.

\section{Resultados}

Los resultados aquí presentados se basan rigurosamente en la metodología expuesta. Queda completamente definido el Elemento Prismático Paramétrico Tridimensional EPPT, incluyendo la metodología para el cálculo de sus áreas, volumen y sus longitudes. Asimismo, se plantearon los sistemas de referencia para la utilización de estos elementos.

También se presentan como resultado algunas pantallas de la herramienta realizada en la Facultad de Ingeniería de la UADY cuyo objetivo es la Edificación Virtual (figura 12). Cabe mencionar que esta herramienta fue construida utilizando conceptos de ingeniería de software y tecnología basada en componentes NET. Entre los componentes más destacados éste nos permite manipular y crear dibujos CAD en 2 y 3 dimensiones, totalmente orientado a objetos. (VectorDraw, 2008). La herramienta fue programada en Visual Studio 6.0 Service Pack 5 (Microsoft, 1998).

\section{Discusión}

Se ha presentado en este artículo el desarrollo de un Elemento Prismático Paramétrico Tridimensional (EPPT) para su uso en la construcción virtual. El lector podrá formularse algunas interrogantes acerca de los planteamientos realizados. Por ejemplo, ¿Por qué solamente se considera en la proyección en planta al EPPT de manera ortogonal?, ¿Acaso esto no llevaría a que las uniones con otros elementos quedasen no de-

finidas adecuadamente? La respuesta a estos inconvenientes que se pueden presentar, se lograrán con las técnicas de inteligencia artificial del software donde sean programados y utilizados estos elementos. Por ejemplo, con lógica difusa o Fuzzy Logic (Coupland et al., 2006) se pueden determinar las proyecciones necesarias del elemento para cerrar las uniones entre uno o más EPPTs cuando éstas sean requeridas (figura 13). Pero sin alterar la manera en que el usuario los ha definido para su uso. Cabe aclarar que estás técnicas están fuera del alcance de este artículo. 


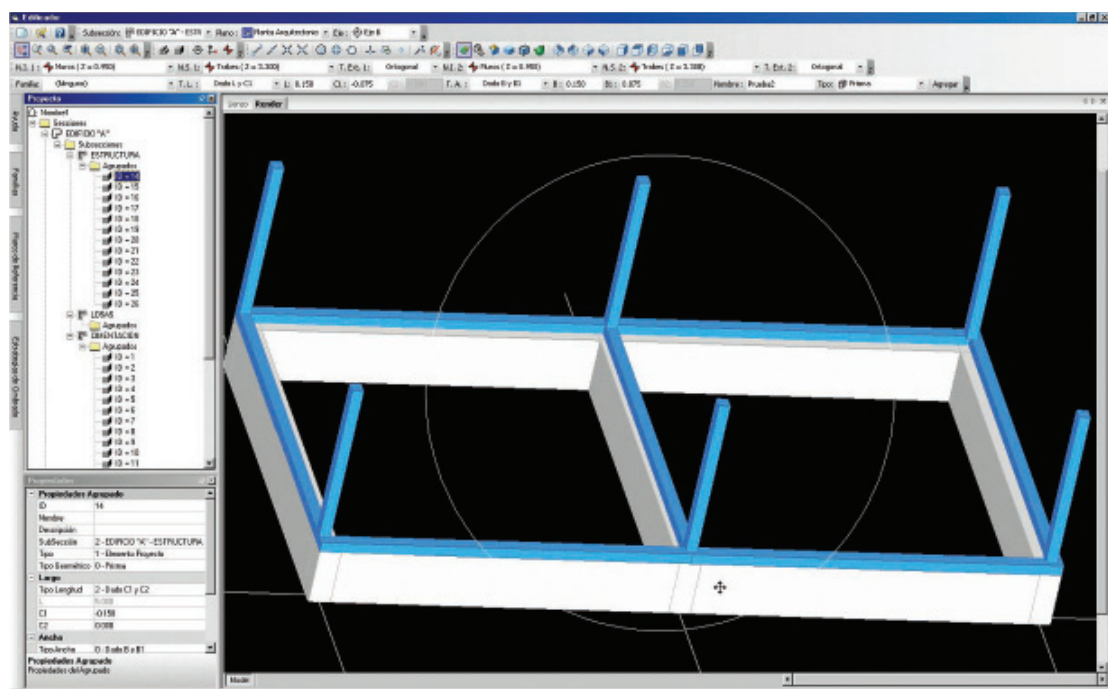

Figura 12 (continuación). Pantallas de la herramienta desarrollada aplicando los EPPT's

Por otro lado, los EPPT no resuelven todos los casos de edificación virtual que existen. Por ejemplo, una losa o un piso pudieran tener más de cuatro vértices en proyección en planta (figura 14). Esto requeriría otro tipo de elemento para el cual habría que hacer un desarrollo específico.

Otro tipo de elementos a desarrollar pudieran ser los requeridos para construir las instalaciones hidráulicas, sanitarias, eléctricas, voz y datos, etc.

\section{Conclusiones}

Existen algunos paquetes de software comerciales a nivel mundial cuyo objetivo es la edificación virtual. Sin embargo, la mayoría fun-

Los puntos P4 y P8 del EPPT2 en (a) son sustituidos por P4' y P8' en (b) mediante el cálculo de sus intersecciones con el EPPT1 automáticamente con técnicas de inteligencia artificial

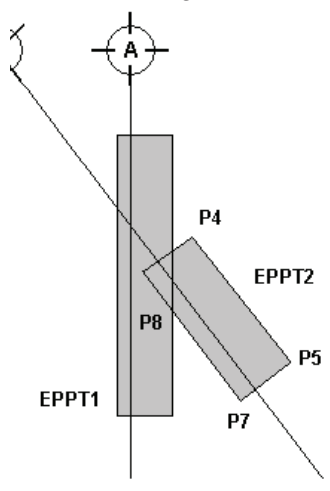

(a)

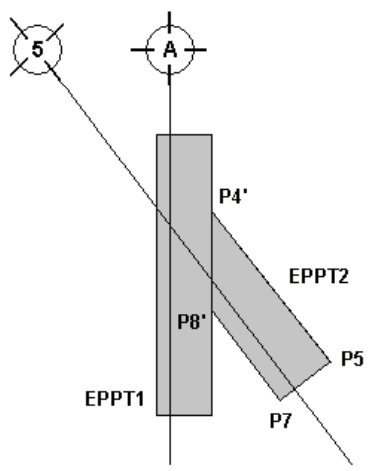

(b)
Figura 13. Unión entre dos EPPT resueltas con técnicas de inteligencia artifical

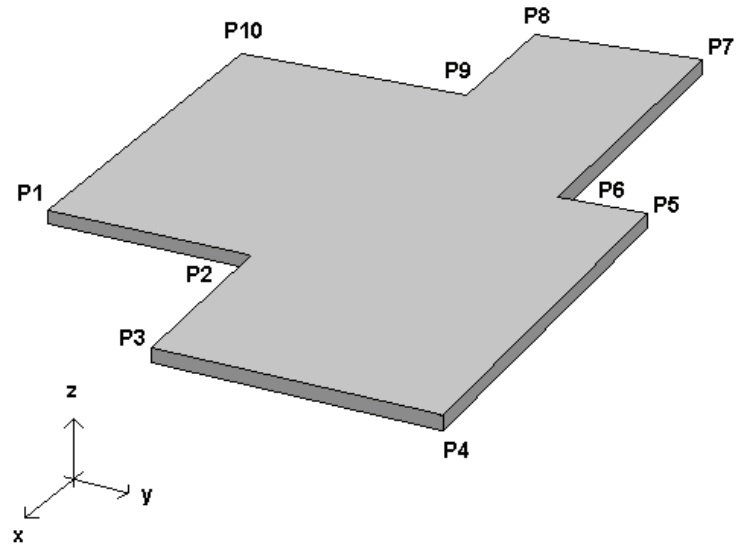

Figura 14. Elemento paramétrico tridimensional para losa o piso cionan como cajas negras que impiden ver cómo son definidos los elementos utilizados. Por lo que esos paquetes no aportan bases para el desarrollo de su mejora por razones obviamente comerciales y de secreto industrial. Sin embargo, en este artículo se presenta de manera detallada el desarrollo de un tipo específico de elemento que puede ser utilizado para la edificación virtual. Que puede resolver una parte significativa de los casos que se presentan en la construcción tales como: muros, columnas, zapatas, dados, dalas, cadenas, muretes, mochetas, etc. Por nombrar algunos de ellos.

La edificación virtual que se propone utilizar estos elementos queda perfectamente definida mediante un sistema de ejes constructivos en planta y un sistema de niveles en alzado. Esto es de vital importancia para revisar la ubicación exacta de algún elemento, sobre todo para fines de cuantificación.

Las matemáticas utilizadas para desarrollar los conceptos aquí expuestos son de un nivel accesible para el público lector, puesto que se usan varios tópicos del álgebra vectorial que por lo general son del dominio del público lector de revistas para la construcción o de ingeniería.

Asimismo, puede ser una herramienta para algún curso de matemáticas, de alguna licenciatura en construcción o ingeniería civil, utilizándolo como material didáctico para el estímulo de los estudiantes al posible uso de los conceptos matemáticos aprendidos para el mejor desarrollo de herramientas tecnológicas actuales. 


\section{Referencias}

AutoDesk, Revit, www.autedesk.com, 2009.

Chen P.H., Cui L., Yang Q., Ting S., Tiong R. Implementation of IFC-Based Web Server for Collaborative Building Design Between Architects and Structural Engineers. Automation in Construction 14:115-128, 2005.

Coupland S., John R. Geometric Type-1 and Type-2 Fuzzy Logic Systems. IEEE Transactions on Fuzzy Systems, pp. 1-12. 2006.

EcoSoft, AllPlan, www.ecosoft.com.mx, 2009.

Graphisfot, ArchiCAD www.graphisoft.com, 2009.

Kunigahalli R. 3D Modeling for Computer-Integrated Construction of RC Structures. J. Comp. in Civ. Engrg.ASCE, 11(2)92101. 1997.
Nassar K., Thabet W., Beliveau Y. Building Assembly Detailing Using Constraint-Based Modeling. Automation in Construction, 12:365-379. 2003

NeoData, NeoDataCAD, www.neodata.com.mx, 2009.

Sacks R., Eastman Ch., Lee G. Parametric 3D Modeling in Building Construction with Examples from Precast Concrete. Automation in Construction, 13:291-312, 2004.

Suter G., Brunner K., Mahdavi A. Building Model Reconstruction Based on Sensed Object Location Information. Automation in Construction, 16:2-12.2007.

Talbot K. Context-Sensivity and Visual Engagement in Generative Systems. Automation in Construction, 16:54-60, 2007.

VectorDraw, www.vdraw.com, 2008.

Visual Studio 6.0 Service Pack 5, Microsoft, 1998.

\section{Semblanza de los autores}

Jesús Nicolás Zaragoza-Grifé. Recibió el grado de ingeniero civil en 1997 y el de maestro en ingeniería-construcción en 2001, ambos por la Facultad de Ingeniería de la Universidad Autónoma de Yucatán. De 2003 a la fecha, ha impartido cursos en las áreas de ciencias básicas, construcción e informática en la maestría en ingeniería, así como en las licenciaturas de ingeniería civil y diversos cursos de educación continua en la UADY. Cuenta con 8 años de experiencia laboral y 6 como director de una empresa consultora de tecnologías de información para control de proyectos para empresas constructoras. Actualmente es profesor investigador titular " $\mathrm{A}$ " en la Facultad de Ingeniería de la UADY.

Josué Gerardo Pech-Pérez. Recibió el grado de ingeniero civil en 1983 y el de maestro en ingeniería-construcción en 1996, ambos por la Facultad de Ingeniería de la Universidad Autónoma de Yucatán. De 1983 a la fecha, ha impartido cursos en las áreas de construcción e informática en la maestría en ingeniería, en las licenciaturas de ingeniería civil y arquitectura, así como diversos cursos de educación continua en la UADY. Cuenta con 10 años de experiencia laboral y 20 en consultoría en informática en diversas empresas constructoras. Actualmente es profesor de carrera titular " $C$ " en la Facultad de Ingeniería de la UADY. 